\title{
СПЕЦИФИЧЕСКИЕ ОСОБЕННОСТИ ДИСТРЕССА У ПАР, ПРИМЕНЯЮЩИХ ПРЕИМПЛАНТАЦИОННУЮ ГЕНЕТИЧЕСКУЮ ДИАГНОСТИКУ В КАЧЕСТВЕ ВСПОМОГАТЕЛЬНОЙ РЕПРОДУКТИВНОЙ ТЕХНОЛОГИИ, И ВОЗМОЖНЫЕ СТРАТЕГИИ ПСИХОЛОГИЧЕСКОЙ РАБОТЫ С ТАКИМИ ПАРАМИ ${ }^{1}$
}

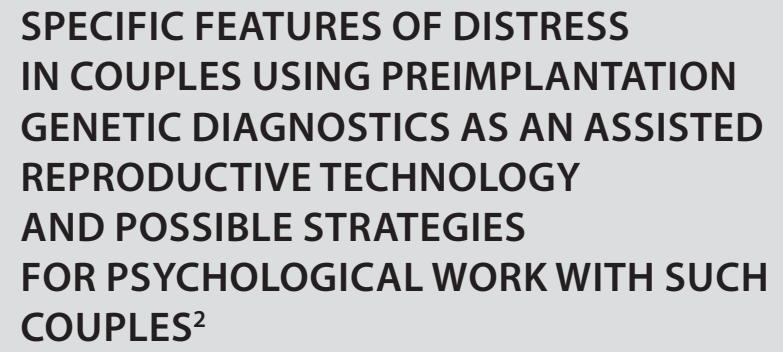

SPECIFIC FEATURES OF DISTRESS IN COUPLES USING PREIMPLANTATION GENETIC DIAGNOSTICS AS AN ASSISTED REPRODUCTIVE TECHNOLOGY AND POSSIBLE STRATEGIES FOR PSYCHOLOGICAL WORK WITH SUCH COUPLES $^{2}$

\section{A. Zufman}

Summary: The article reviews studies on the specifics of psychological responses to the use of preimplantation genetic diagnostics (PDG) in patients and their partners, in particular, significant differences between the experience of passing through $P G D$ and the experience of using other reproductive technologies (for example, in vitro fertilization). The specificities of experiences of stress in couples who choose PGD will ensure quality psychological support such couples at all stages of treatment to predict the individual risk of distress and related mental complications, to improve the efficiency of the process of medical information to potential patients, to avoid acute and delayed stress reaction, and in the future to improve the mental health of the citizens, faced with infertility and the risk of having children with severe chromosomal and genetic diseases.

Keywords: preimplantation genetic diagnostics, assisted reproductive technologies, miscarriage, psychological well-being of mothers, decisionmaking stress, in vitro fertilization.
Зуфман Анна Ивановна

Преподаватель, Российская Академия народного хозяйства и государственной службы при Президенте РФ anivzu@yandex.ru

Аннотация: Статья посвящена обзору исследований, посвященных специфике психологических реакций на применение преимплантационной генетической диагностики (ПДГ) у пациенток и их партнеров, в частности - существенным различиям между опытом прохождения через ПГД и опытом применения других репродуктивных технологий (например, экстракорпорального оплодотворения). Изучение специфических особенностей переживания стресса у пар, выбирающих ПГД, позволит обеспечить качественное психологическое сопровождение таких пар на всех этапах выбора лечения, прогнозировать уровень индивидуальных рисков возникновения дистресса и сопутствующих психических осложнений, повысить эффективность процесса медицинского информирования потенциальных пациентов, избежать острых и отложенных стрессовых реакция, а в перспективе улучшить показатели психического здоровья у граждан, столкнувшихся с бесплодием и риском рождения детей с тяжелыми хромосомными и генетическими заболеваниями.

Ключевые слова: преимплантационная генетическая диагностика, вспомогательные репродуктивные технологии, невынашивание беременности, психологическое благополучие матерей, стресс принятия решений, экстракорпоральное оплодотворение.
$\Pi$ реимплантационная генетическая диагностика (ПГД) - это вспомогательная репродуктивная технология, эффективно используемая с 90-х годов прошлого века для помощи парам с различными репродуктивными проблемами и/или наличием в анамнезе генетических заболеваний. На начальных этапах основным показанием для ее применения был риск развития у будущего ребенка тяжелых наследственных заболеваний, проявляющихся в раннем возрасте, таких как болезнь Тея-Сакса, кистозный фиброз или спинальная мышечная атрофия [21]. Однако с развитием технологий диапазон показаний к применению ПгД был существенно расширен и на данный момент эта технология используется в том числе для скрининга различных анеуплоидий или определения HLA-совместимости, а также для помощи матерям и парам, страдающим от привычной невынашиваемости [11]

Вслед за научными и клиническими разработками в этой области, начались исследования психологическо- 
го и психосоциального влияния ПГД на пациентов. Сама процедура и процесс принятия решения о ее прохождении часто вызывают психологическое напряжение, оказывая влияние и на самих матерей, и на их семьи [8, $81,24]$. Возможность применения генетической диагностики эмбриона с последующим отбором и подселением в матку часто рассматривается как «последний шанс» родить здорового ребенка, и может восприниматься женщиной и ее партнером как спасительный выход, но одновременно - как «приговор», поэтому само принятие решения о подобном виде вмешательства становится стрессом, особенно - для тех, кто перед этим пережил опыт неизлечимого бесплодия, хронического невынашивания, потерь беременности или рождения детей с тяжелыми генетическими и хромосомными нарушениями. Кроме того, специфические особенности осуществления протокола ПГД с последующим отбором наиболее здоровых и жизнеспособных эмбрионов, сопровождается стрессом и требует от женщины или от пары принятия достаточно тяжелых с психологической точки зрения решений. Все это говорит о том, что пациенты, прибегающие к преимплантационной генетической диагностике в качестве вспомогательной репродуктивной технологии, нуждаются в качественном психологическом консультировании на этапе принятия решения, а также в психологическом сопровождении на всех этапах осуществления прокола. Предыдущие исследования пар, обращавшихся к помощи вспомогательных репродуктивных технологий, таких как ПГД и ЭКО, продемонстрировали, что эффекты, оказываемые этими факторами на психическое здоровье пар, сохраняются на протяжении трех лет [14].

Многие исследователи подчеркивают нехватку исследований, посвященных влиянию применения ПгД на пациентов и опыту переживания связанного с этим типом диагностики стресса $[8,15,18,22]$. Во многом, это связано с тем, что эта технология появилась не так давно, и доступна далеко не всем. Кроме того, этическая неоднозначность применения данной технологии создает дополнительные сложности в организации исследований. Однако некоторые выводы можно сделать уже сейчас, частично - опираясь на многочисленные исследования, посвященные пациентам, проходящих через процедуру экстракорпорального оплодотворения (ЭКО), влияние которой на психическое здоровье матерей и их партнеров изучено гораздо лучше (процедура ЭКО применяется по всему миру намного дольше, доступнее для населения). Протокол ПГД включает в себя экстракорпоральное оплодотворение, поэтому с технической точки зрения эти технологии похожи: после стимуляции овуляции осуществляется забор яйцеклеток женщины, оплодотворение их происходит экстракорпорально, после чего несколько наиболее «перспективных» эмбрионов подсаживаются в матку. С точки зрения инвазивности и воздействия на организм женщины практически нет разницы, используется ли просто ЭКО или ЭКО вместе с ПГД, различие заключается лишь в способе отбора эмбрионов: в случае обычного ЭКО отбор не происходит или осуществляется с помощью визуальной оценки материала врачом, а при применении протоколов ПГД специалисты проводят генетический анализ каждого эмбриона, чтобы исключить наличие хромосомных мутаций и наследственных заболеваний. Но именно эти различия существенно меняют психологическое восприятие женщиной процедуры отбора. Согласно статистике, сами пациенты, обращающиеся в клиники для прохождения ПГД, обладают рядом ключевых отличий от тех, кто прибегает к обычному ЭКО. В частности, к ПГД зачастую прибегают пары с наличием в личном или семейном анамнезе генетических заболеваний, которые сами по себе являются источником дистресса. Таким образом, применение техник психологической поддержки, разработанных на основании результатов исследования пациентов, прошедших ЭКО, недостаточно для помощи пациентам, выбравшим ПГД [16].

Следует учитывать, что сам процесс принятия решения об использовании подобных вспомогательных репродуктивных технологий является стрессовым. Индивидуально или как пара, пациенты вынуждены рассматривать моральные и этические вопросы, касающиеся ценности жизни будущего ребенка, который может унаследовать генетическое заболевание. Восприятие этого факта во многом различается у тех пар, которые уже имеют одного или нескольких детей с тяжелыми хромосомными или генетическими заболеваниями, и у тех, кто впервые столкнулся с подобным риском. Кроме того, парам или матерям приходится взвешивать потенциальные медицинские и финансовые риски, связанные с процедурой ЭКО, риски, связанные с техническими ограничениями процедуры, к примеру, неточными результатами тестирования эмбрионов [11]; рассматривать вариант повторного использования ПГТ, в случае, если первая попытка окажется неудачной [6]. Все эти факторы, в сочетании с наличием психологических проблем, вызванных бесплодием, привычным невынашиванием или наличием в личном или семейном анамнезе наследственных заболеваний, могут быть причиной дистресса и неуверенности у пар еще до обращения в клинику [5].

Ретроспективное исследование опыта женщин, прошедших ПГД, проведенное в 2005 году, демонстрирует еще один фактор формирования дистресса и неуверенности - взаимодействие с системой здравоохранения и медицинским персоналом [15]. Так, 40\% респондентов считают, что в ходе лечения они чрезмерно доверяли своим врачам. Одна пациентка вспоминала, что в ходе лечения она старалась задавать лечащим врачам как можно меньше вопросов, чтобы ее не воспринимали как трудного пациента. Часть пациенток полагала, что вместе с ПГД им придется проходить и ПНД (пренатальную 
генетическую диагностику) тоже. По словам одной из респонденток, ее консультант-генетик сообщил ей о том, что ей необходимо в дополнение к ПГД пройти процедуру биопсии хориона (CVS), однако руководство клиники не знало о том, что на пациентов оказывается подобное давление со стороны их сотрудников [16].

Исследование родителей, проведенное в Бельгии в 2008 году [20], продемонстрировало отсутствие значимых различий в уровне родительского стресса между тремя группами респондентов: первые две группы использовали ПГД и ЭКО соответственно, родители из третьей группы не прибегали к помощи вспомогательных репродуктивных технологий для зачатия. Согласно данным заполненных респондентами опросников, во всех группах приблизительно одинаковое количество мужчин и женщин оценивали уровень стресса как низкий, умеренный и высокий. Баллы по опроснику общего состояния здоровья, который был использован для выявления и/или измерения уровня депрессии, тревоги, соматических проявлений дистресса и социальной изоляции, также существенно не различались между респондентами из разных групп.

Однако, у пар, которым приходится прибегать к преимплантационной генетической диагностике эмбрионов, существует ряд существенных отличий, которые нельзя не учитывать при планировании психологической помощи таким людям [16]. Многие исследования пар, использовавших ПГД в прошлом или проходящих процедуру на момент исследования, включают анкетирование, в ходе которого респондентам предлагается выбрать преимущества и недостатки этой технологии из предложенного списка или же указать их самим, а также изложить общее отношение к ПГД. В качестве преимуществ наиболее часто указывались: возможность родить здорового ребенка, отсутствие необходимости в прерывании беременности как части процедуры, и значительное снижение уровня стресса и тревоги в сравнении с пренатальной диагностикой, которая при определенных результатах тестирования может закончиться абортом $[7,12,24]$. В этом смысле, при выборе между ПНД и ПГД, мы опираемся на то, что второй вариант предполагает меньше стресса для женщины за счет того, что отбор производится до подселения эмбрионов в матку, а значит, не влечет за собой риск аборта на относительно позднем сроке. Однако, для определения особенностей дистресса этих пар, нам стоит рассмотреть и указанные ими недостатки ПГД. Так, в их числе: риск повреждения эмбриона в ходе тестирования, необходимость прохождения ЭКО, относительно высокий риск неудачи при прохождении процедуры (беременность может не состояться), необходимость длительного пребывания в списке ожидания и стоимость процедуры, а также сопутствующие моральные переживания о судьбе неиспользованных эмбрионов [16].
В ходе осуществления протокола ПДГ не все полученные путем ЭКО эмбрионы переносятся в матку женщины. Часть из них отбраковывается из-за обнаружения хромосомных патологий, генетических и наследственных заболеваний. Часть эмбрионов, признанных после генетического анализа здоровыми, тоже не используются. Принятие решения о дальнейшей судьбе неиспользованных эмбрионов - это относительно изученный и задокументированный процесс. Выявлено, что разные пары по-разному относятся к тому, что отбракованные эмбрионы никогда не будут перемещены в матку и никогда не получат шанс родиться. Многие пары, у которых уже есть ребенок, страдающий тем или иным наследственным или генетическим заболеванием, зачастую проводят параллели между ним и эмбрионами, полученными в ходе ПГД, что создает дополнительный стресс в ходе принятия решения об их утилизации. Другие же, хоть и говорят об эмбрионах как о своих потенциальных нерожденных детях (а некоторые - как об уже рожденных), все же готовы отдать их другим бесплодным парам или утилизировать. Стоит отметить, что подверженные генетическим заболеваниям эмбрионы не могут участвовать в программе донорства эмбрионов, однако родители могут дать согласие на их исследование в научных целях $[5]$.

Для некоторых пар необходимость уничтожения поврежденных эмбрионов или здоровых эмбрионов, которые не используются для подселения в матку, делает всю процедуру ПГД этически запутанной. Некоторые матери воспринимают необходимость принятия решения в данном аспекте как выбор между «убийством» и обречением больного ребенка на мучительную жизнь с неизлечимым заболеванием, что создает дополнительный дистресс.

Среди прочих, стоит отметить этнографическое исследование, проведенное в Великобритании в 2004 году Робертсом и Франклином [23]. Несмотря на скромную выборку (21 человек), результаты этого исследования позволяют посмотреть на проблемы пользователей Пгд под другим углом. Так, в числе прочего, было обнаружено, что одним из факторов, формирующих дистресс и являющихся причиной социальной изоляции является медийная интерпретация ПГД. Во многих средствах массовой информации эта процедура представляется как способ создания "дизайнерских младенцев" - детей с набором заранее выбранных родителями генетических характеристик. Аналогичную реакцию у респондентов вызывает распространенное мнение о необходимости более жесткого юридического регулирования ПГД для предотвращения использования этой технологии в евгенических целях. Во многих случаях пары, проходящие через ПГД, сталкиваются с социальной изоляцией: учитывая условную уникальность такого состояния, пары не могут в полной мере получить психологическую под- 
держку от собственных друзей или родственников, оставаясь, таким образом, наедине с проблемой [16]. Часть матерей сталкиваются с непониманием и осуждением со стороны собственных родственников и близких друзей, некоторые испытывают сильный стыд из-за того, что прибегают к подобным решениям, и вынуждены скрывать от окружающих, через что они проходят. Часто попытки объяснить близкому окружению, что такое ПГД, наталкиваются на препятствия, связанные с низким уровнем медицинской грамотности, что тоже приводит к тому, что пациентам проще скрывать особенности способа зачатия, нежели проводить дополнительную работу по информированию. При составлении протоколов психологического сопровождения родителей, прибегающих к ПГД, необходимо учитывать тот факт, что многие из них страдают от такого рода стигматизации, а также подвергаются давлению со стороны общества. Кроме того, специалисты, оказывающие информационную и психологическую поддержку таким парам, должны избегать любых интервенций, которые могут быть восприняты пациентами как осуждение их выбора, так как люди, подвергающиеся стрессу принятия решений, особенно чувствительны к такого рода ошибкам.

Сам процесс прохождения процедуры ПГД также является тяжелым для пациентов: как физически, так и психологически. В исследовании, проведенном в 2002 году с участием 36 пар из Великобритании и Испании, которые ранее уже использовали ПГД для зачатия ребенка, рассматривался стресс, который они испытывали в ходе процедуры сравнении со стрессом при ПНД [19]. Большая часть респондентов (41\%) описали стресс как «очень сильный», при этом двое особо отметили, что это было самое стрессовое событие в их жизни. 35\% и 23\% респондентов охарактеризовали стресс при ПГД как «умеренный» и «легкий» соответственно. Самым тяжелым периодом при прохождении ПГД большая часть пар посчитала время между имплантацией эмбриона в матку и уведомлением о результатах теста на беременность. Треть пар заявила, что этот процесс в целом негативно сказался на их отношениях. Из 36 пар 20 ранее проходили процедуру пренатальной диагностики и большая часть из них (40\% или 8 пар) описали свой опыт прохождения ПНД как менее стрессовый в сравнении с ПГД, меньшая же часть респондентов (35\% или 7 пар) дала противоположный ответ. Исходя из этого, мы можем предположить, что консультирование матерей и их партнеров необходимо осуществлять на всех этапах прохождения процедуры, начиная от принятия решения об использовании ПГД в качестве вспомогательной репродуктивной технологии, и до получения положительного результата теста на беременность. При этом с специалист должен сосредотачиваться на таких аспектах, как стресс принятия решения, беспокойство о судьбе эмбрионов, страх неудачи, тревоги, связанные с желанием родить здорового ребенка, стрессы, порожденные социальной изоляцией и общественным осуждением, а также риски распада семьи в ходе прохождения через все этапы процедуры.

Практически на сегодняшний день не существует исследований по изучению привязанности к эмбриону и ребенку, рожденному после ПГД, хотя они также могут быть очень информативными. Задокументированные мнения женщин о системах психологической поддержки и дальнейшей судьбе неиспользованных эмбрионов позволят поставщикам медицинских услуг улучшить обслуживание будущих клиентов, которые вынуждены пройти этот тяжелый путь в надежде на рождение здорового ребенка. Понимание информационных потребностей пациентов также поможет клиникам облегчить этот путь для них.

А.Л. Грейл [9] в своем обзоре исследований психологического влияния бесплодия поднял актуальные здесь вопросы. Выводы этой статьи аналогичны более позднему обзору С.М. Верхаака и его коллег[25], написанному в 2007 году: исследования, посвященные психопатологии, не выявили серьезных различий между группой испытуемых с бесплодием и контрольной группой. Грейл утверждает, что существующий в настоящее время подход к подобным исследованиям с медицинской, а не психосоциальной точки зрения, является ошибочным. Большая часть исследований была посвящена сравнению уровня дистресса, который испытывают пары при ЭКО, с аналогичным показателем других групп людей, обычно избегая нулевых результатов, и не обращали внимание на то, как бесплодие и его лечение влияют на человека. Поэтому для будущих исследователей может быть полезным изучить дистресс с точки зрения отдельного человека, а не группы респондентов $[9,13]$.

На сегодняшний день мы имеем дело в основном с исследованиями, проведенными в других странах, без учета специфических особенностей российской выборки, в то время как реакция российских пар на стресс, связанный с принятием этически неоднозначных решений, может существенно отличаться от того, как подобный опыт переживается партнерами в других государствах. Так, согласно исследованиям, проведенным Разваляевой А.Ю. в 2018 году, способность преодолевать стресс, связанный с принятием решений и ситуациями неопределенности, у российских пар существенно зависит от уровня образования, что является специфической особенностью российской выборки [3]. Кроме того, можно предположить, что отношение к беременности, родам, проблемам материнства и бесплодия, в России тоже значимо отличается от общемировых показателей [1]. В частности, доказано, что в общественном сознании бесплодие часто воспринимается как социальная, психологическая и нравственная проблема, а не как медицинская, поэтому реакция общества и отдельных инди- 
видов на медицинские способы ее решения может быть искаженной. Кроме того, как показывают исследования, применение вспомогательных препродуктивных технологий, в особенности, связанных с экстракорпоральным оплодотворением и с генетической диагностикой, зачастую воспринимаются в общественном сознании как угроза «естественному порядку вещей», «семейным ценностям», «духовности» и так далее [2], что тоже влияет на специфику потенциального консультирования именно российских матерей.

Тем не менее, уже на данном этапе мы можем ут- верждать, что психологические риски у матерей и их партнеров при прохождении процедуры ПГД в качестве вспомогательной репродуктивной технологии достаточно высоки, а влияние ПГД на психическое благополучие пациентов и их семей, при всем внешнем сходстве процедур, существенно отличается от того влияния, которое оказывает применение обычного экстракорпорального оплодотворения, что необходимо учитывать при разбработке программ психологического сопровождения пар в процессе осуществления ПГД, а также возможной последующей психологической коррекции вызванных этим опытом расстройств и нарушений адаптации.

\section{ЛИТЕРАТУРА}

1. Григорьева Наталия Сергеевна, Куштанина Вероника Александровна, Чубарова Татьяна Владимировна, Яковлева Ирина Викторовна Бездетность в России: социологическое измерение // Народонаселение. 2013. №4 (62)

2. Емельянова Татьяна Петровна, Мишарина Анна Владимировна, Вопилова Ирина Евгеньевна Репрезентация рисков применения вспомогательных репродуктивных технологий // Горизонты гуманитарного знания. 2019. №4.

3. Разваляева А.Ю. Апробация опросника «Тенденции в принятии решений» на русскоязычной выборке // Консультативная психология и психотерапия. 2018. T. 26. № 3. C. 146-163. doi: 10.17759/срp.2018260308

4. Banerjee, I., Shevlin, M., Taranissi, M., et al., 2008. Health of children conceived after preimplantation genetic diagnosis: a preliminary outcome study. Reprod. BioMed. Online 16, 376-381.

5. Cunningham J., Goldsmith L., Skirton H. The evidence base regarding the experiences of and attitudes to preimplantation genetic diagnosis in prospective parents // Midwifery. 2015. Vol. 31 (2). P. 288-296

6. Derks-Smeets IA, Gietel-Habets JJ, Tibben A, Tjan-Heijnen VC, Meijer-Hoogeveen M, Geraedts JP, van Golde R, Gomez-Garcia E, vanden Bogaart E, van Hooijdonk Met al.Decision-making on preimplantation genetic diagnosis and prenatal diagnosis: a challenge for couples with hereditary breast and ovarian cancer.Hum Reprod 2014;29:1103-1112.

7. Farra, C., Nassar, A.H., Usta, I.M., et al., 2008. Acceptance of preimplantation genetic diagnosis for beta-thalassemia in Lebanese women with previously affected children. Prenat. Diagn.28, 828-832.

8. Galjaard, H., 2003. Report of the IBC on the Preimplantation Genetic Diagnosis and Germ-line Intervention. United Nations Educational, Scientific and Cultural Organization, Paris.

9. Greil, A.L., 1997. Infertility and psychological distress: a critical review of the literature. Soc. Sci. Med. 45, 1679-1704.

10. Hammarberg, K., Fisher, J.R., Wynter, K.H., 2008. Psychological And social aspects of pregnancy, childbirth and early parenting after assisted conception: a systematic review. Hum. Reprod.Update 14, 395-414

11. Harper JC, Aittomäki K, Borry P, Cornel MC, de Wert G, Dondorp W, Geraedts J, Gianaroli L, Ketterson K, Liebaers let al.Recent developments in genetics and medically-assisted reproduction: from research to clinical applications. Hum Reprod Open 2017;107.

12. Hui, P.W., Lam, Y.H., Chen, M., et al., 2002. Attitude of at-risk subjects towards preimplantation genetic diagnosis of alpha- and beta-thalassaemias in Hong Kong. Prenat. Diagn. 22, 508-511.

13. Jacob, M.C., McQuillan, J., Greil, A.L., 2007. Psychological distress by type of fertility barrier. Hum. Reprod. 22, 885-894.

14. Järvholm S, Thurin-Kjellberg A, Broberg M. Experiences of preimplantation genetic diagnosis (PGD) in Sweden: a three-year follow-up of men and women. J Genet Couns 2017;26:1008-1016.

15. Kalfoglou, A.L., Scott, J., Hudson, K., 2005. PGD patients' andproviders' attitudes to the use and regulation of preimplantationgenetic diagnosis. Reprod. BioMed. Online 11, 486-496

16. Karatas J.C., Strong K.A., Barlow-Stewart K. [et al.] Psychological impact of preimplantation genetic diagnosis: a review of the literature // Reprod. Biomed. Online. 2010. Vol. 20 (1). P. 83-91

17. Katz, M.G., Fitzgerald, L., Bankier, A., et al., 2002. Issues and concerns of couples presenting for preimplantation genetic diagnosis (PGD). Prenat. Diagn. 22, $1117-1122$.

18. Knoppers, B.M., Bordet, S., Isasi, R.M., 2006. Preimplantation Genetic diagnosis: an overview of socio-ethical and legal considerations. Annu. Rev. Genomics Hum. Genet. 7, 201-221.

19. Lavery, S., Aurell, R., Turner, C., et al., 2002. Preimplantationgenetic diagnosis: patients' experiences and attitudes. Hum.Reprod. 17, 2464-2467

20. Nekkebroeck, J., Bonduelle, M., Desmyttere, S., et al., 2008. Socio-emotional and language development of 2-year-old chil-dren born after PGD, PGS, and parental well-being. Hum.Reprod. 23, 1849-1857.

21. Offit, K., Kohut, K., Clagett, B., et al., 2006. Cancer genetic testing and assisted reproduction. J. Clin. Oncol. 24, 4775-4782. 
22. Pastore L.M., Cordeiro Mitchell C.N., Rubin L.R., Nicoloro-SantaBarbara J., Genoff Garzon M.C., Lobel M., Patients' preimplantation genetic testing decision-making experience: an opinion on related psychological frameworks, Human Reproduction Open, Volume 2019, Issue 4, 2019

23. Roberts, C., Franklin, S., 2004. Experiencing new forms of genetic choice: findings from an ethnographic study of preimplantation genetic diagnosis. Human Fertility 7, 285-293.

24. Soini, S., Ibarreta, D., Anastasiadou, V., et al., 2006. The interface between assisted reproductive technologies and genetics: technical, social, ethical and legal issues. Eur. J. Hum. Genet. 14,588-645.

25. Verhaak, C.M., Smeenk, J.M., Evers, A.W., et al., 2007. Women's emotional adjustment to IVF: a systematic review of 25 years of research. Hum. Reprod. Update 13, $27-36$.

(c) Зуфман Анна Ивановна (anivzu@yandex.ru).

Журнал «Современная наука: актуальные проблемы теории и практики»

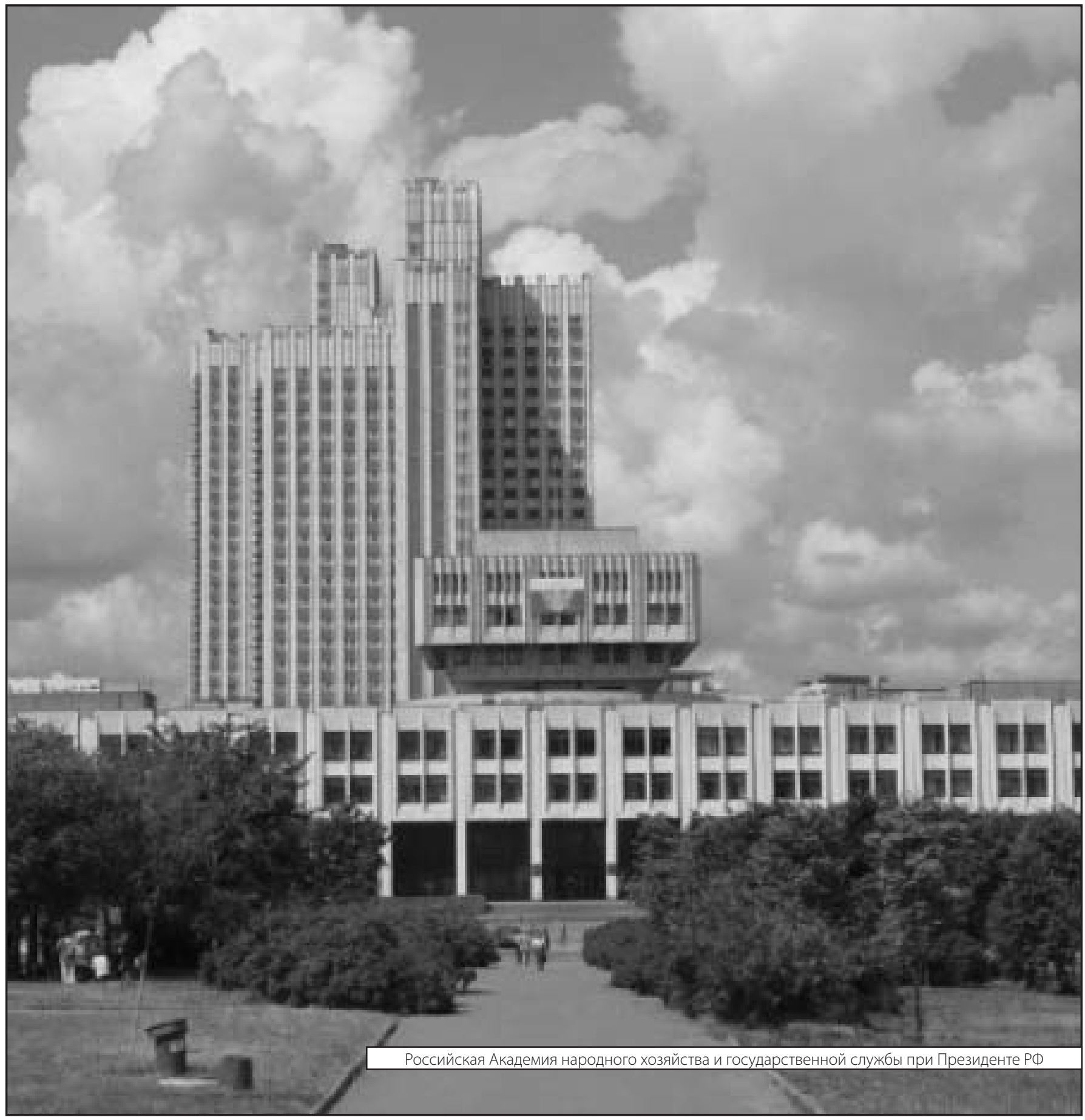

